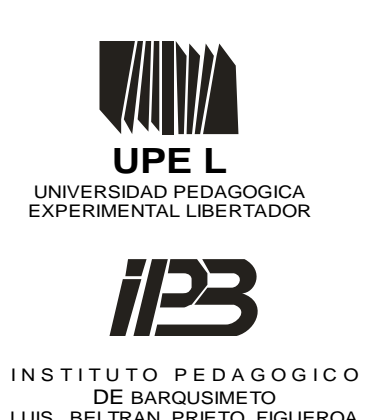

DE BARQUSIMETO
LUIS BELTRAN PRIETO FIGUEROA

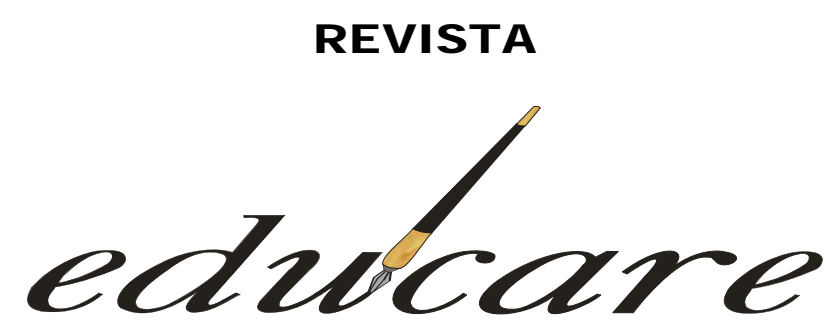

Órgano Divulgativo de la Subdirección de Investigación y Postgrado del Instituto Pedagógico de Barquisimeto “Luis Beltrán Prieto

Figueroa"

BARQUISIMETO - EDO. LARA - VENEZUELA

NUEVA ETAPA

FORMATO ELECTRÒNICO

DEPOSITO LEGAL: ppi201002LA3674

Volumen $17 \mathrm{~N}^{\circ} 3$

ISSN: 2244-7296

Septiembre-Diciembre 2013

\title{
TEORÍAS IMPLÍCITAS DE LA CREATIVIDAD CONSTRUIDAS POR DOCENTES DE DIDÁCTICA DE LA UPEL-IPB
}

\section{IMPLICIT THEORIES BUILT BY THE DIDACTICS TEACHERS FROM THE UPEL-IPB}

\section{Pablo Antonio Carmona Pérez Universidad Pedagógica Experimental Libertador Instituto Pedagógico de Barquisimeto \\ Venezuela}




\section{TEORÍAS IMPLÍCITAS DE LA CREATIVIDAD CONSTRUIDAS POR DOCENTES DE DIDÁCTICA DE LA UPEL-IPB}

\section{IMPLICIT THEORIES BUILT BY THE DIDACTICS TEACHERS FROM THE UPEL-IPB}

\section{INVESTIGACION}

Recibido: 18-10-13

\author{
Pablo Antonio Carmona Pérez ${ }^{*}$ \\ UPEL-IPB \\ Venezuela \\ Aceptado: 11-12-13
}

\begin{abstract}
RESUMEN
El presente artículo refiere una experiencia de investigación cuyo propósito fue develar las teorías implícitas construidas por los docentes de didáctica del Departamento de Formación Docente UPEL-IPB sobre la creatividad. El trabajo es de naturaleza cualitativa apoyado en el método etnográfico. Los actores sociales comprometidos con la investigación fueron docentes de didáctica de la UPEL-IPB. Las técnicas para la producción de información fueron la observación participante, la entrevista en profundidad y los testimonios focalizados. Los hallazgos revelan cinco (5) teorías implícitas amplias sobre la creatividad, que son: Teoría de la creatividad como proceso, Teoría de la creatividad como ambiente, Teoría de la creatividad como producto, Teoría de la creatividad como persona y Teoría integral de la creatividad. En consecuencia, se puede concluir que el docente de didáctica de la UPEL-IPB ha construido inconscientemente teorías acerca de la creatividad, las cuales son un conjunto de representaciones mentales bien significativas, por cuanto configuran un bagaje de conocimientos valiosos para la comprensión de procesos relacionados con la creatividad, la enseñanza y el aprendizaje.
\end{abstract}

Descriptores: teorías implícitas, docentes de didáctica, creatividad.

\begin{abstract}
The present paper refers to a research experience aiming to uncover the implicit theories about creativity generated by didactics teachers from the Department of Teachers' Formation at the UPELIPB. It was a qualitative research supported by ethnographic methodology. Social actors engaged to the research were didactics teachers from the UPEL-IPB. Techniques for data collection were participant observation, in-depth interviews and targeted testimonies. Findings revealed five (5) wide implicit theories about creativity: theory of creativity as a process, theory of creativity as environment, theory of creativity as a product, theory of creativity as a person and a whole theory of creativity. As a result, it may be concluded that the didactics teacher from the UPEL-IPB has unconsciously built theories about creativity which are a group of meaningful mental representations that shape a baggage of knowledge valuable for the understanding of processes related to creativity, teaching and learning.
\end{abstract}

Keywords: implicit theories, didactic teachers, creativity.

\footnotetext{
${ }^{*}$ Docente adscrito al Departamento de Formación docente de la UPEL-IPB. Magister en Ciencias de la Educación. Barquisimeto. Edo. Lara. Venezuela. E-mail: pavlocarmona@hotmail.com
} 


\section{INTRODUCCIÓN}

El pensamiento del profesor ha sido un tema de gran interés en el campo de la investigación en educación. A partir de los hallazgos obtenidos en los procesos indagatorios, sobre este tema se destacan dos directrices de pensamiento de los profesores. Una de está orientada al conocimiento de las teorías explícitas, también llamadas conscientes, y la otra, referida a las teorías implícitas, inconscientes o tácitas (Porlán y Rivero, 1998).

En relación con las teorías implícitas, éstas han sido construidas por los profesionales de la docencia, inconscientemente, a lo largo del devenir de su práctica en diferentes áreas temáticas o dominios de conocimientos. Además, se conoce que las mismas tienen gran influencia en las acciones que éstos desarrollan en su práctica de enseñanza. Como también se ha pensado acerca de la dificultad que tienen los profesores para expresar sus teorías implícitas de manera coherente y vertebrada. Es por ello que son poco accesibles a la conciencia (Rodrigo, Rodríguez y Marrero, 1993). Estas premisas nos permiten darnos cuenta del amplio campo de exploración de los significados ocultos en el pensamiento de los docentes en una vasta diversidad de dominios de conocimiento y de los retos que tienen las autoridades educativas para para incorporación de estas supuestos implícitos en el debate que se debe generar durante los procesos de transformación de la educación venezolana.

Es imposible desarrollar cambios en las currículas de los diversos niveles educativos si antes no se conoce en estado del pensamiento de los docentes, por cuantos son estos los encargados de operacionalizar las transformaciones, capacitación y actualización en los nuevos enfoques teóricos que se asumirán. De allí que las teorías implícitas se configuran en una de las principales preocupaciones que hoy día se percibe acerca del pensamiento de los docentes.

En tal sentido, se tiene que los docentes construyen estas representaciones mentales en diferentes áreas o dominios del conocimiento y que en muchos de los casos pueden considerarse en construcciones erróneas de las teorías formales. Pero, para efectos de la presente investigación, se creyó conveniente explorar las teorías implícitas de la creatividad, por cuanto ésta se configura en uno de los dominios atientes a la práctica de aula de los profesores que administran los cursos de didáctica del departamento de Formación Docente de la UPEL-IPB.

Revista EDUCARE, Volumen 17, Número 3, Septiembre-Diciembre 2013. DEPOSITO LEGAL: ppi201002LA3674- ISSN: 2244-7296 (Revista Arbitrada e 
Es así como a partir de los planteamientos anteriores cobra sentido el presente estudio, cuyo propósito fue develar las teorías implícitas de los docentes de didáctica del Departamento de Formación Docente, Universidad Pedagógica Experimental Libertador, Instituto Pedagógico de Barquisimeto "Luis Beltrán Prieto Figueroa" (UPEL-IPB), que los mismos han construido sobre la creatividad.

El presente estudio, de naturaleza cualitativa, se caracteriza por la adopción del paradigma de investigación socioconstruccionista y el método etnográfico. Los actores sociales comprometidos con la investigación fueron los docentes adscritos al Departamento de Formación Docente de la UPEL- IPB.

En fin, el artículo quedó estructurado en cuatro (4) secciones. En la primera se destaca la contextualización de la situación de estudio, a través de la descripción del tema y el escenario de la investigación. La segunda contempla el abordaje metodológico asumido durante la investigación. En la tercera se indican los hallazgos más relevantes, reflejados en las categorías develadas. Y la cuarta contiene presentan las reflexiones finales sobre la base de los hallazgos obtenidos.

\section{CONTEXTUALIZACIÓN DEL OBJETO DE ESTUDIO.}

La construcción de teorías implícitas es una función constante de los seres humanos. No sólo de la sociedad occidental o de cualquier otra, sino del científico y hombre común. En esta función se destacan tres dimensiones básicas, que son: la biológica, la social y la representacional. (Rodrigo, Rodríguez y Marrero, ibidem).

En este mismo sentido se pronuncian Argyris y Schön (1978), cuando expresan que:

...los actores sociales (individuos u organizaciones), construyen teorías para orientar sus acciones. El comportamiento que despliegan responde, tanto, a sus teorías (construcciones, esquemas, modelos o mapas mentales) formados por estrategias de acción, supuestos y valores o principios rectores los cuales se manifiestan en los individuos a través de sus conductas, actitudes y creencias...(p.34)

Como se aprecia en la cita anterior, se deja entrever que el comportamiento de las personas está influido por sus representaciones o esquemas mentales. Aspecto que es acuñado por Rodrigo, Rodríguez y Marrero (ob.cit), cuando expresan que las teorías implícitas dirigen las acciones de las personas para responder a las demandas ambientales o del entorno. 
En este mismo sentido, se puede destacar el carácter social de las teorías implícitas. Según Reyes, Salcedo y Perafán (1999), las personas teorizan naturalmente acerca del mundo físico y social que les rodea, sin mostrar un especial interés por la naturaleza propia de la representación en la estructura cognitiva. Debido a que estas teorías constituyen una función cognitiva que proporciona a las personas herramientas para la interpretación y adaptación ante el mundo, la función de éstas puede trascender hasta el punto de comprender, a partir de la misma, otros escenarios constituidos por diferentes personas. Este hecho permitirá intercambios y procesos de interacción, que son comunes en las organizaciones sociales.

En esta misma dirección, Porlán (1996) expresa que las teorías implícitas permiten que los individuos entiendan el mundo a través de ellas, sin importar el análisis que se haga de sí mismas aspecto que dificulta su verbalización. Sin embargo, ello no impide que éstas puedan ser verbalizadas en un momento determinado si las condiciones son propicias.

Asimismo, Bolívar (2000) esgrime que, las teorías implícitas se caracterizan por su imposibilidad de “... traducirse o articularse enteramente en un discurso" (p.156). Ello corrobora el carácter inconsciente de las teorías implícitas, ya que esto supone la imposibilidad de expresar o verbalizar coherentemente este tipo de conocimiento. Lo que implica, según el mismo autor, una manera de saber hacer, más que una manera de saber decir.

Igualmente, frente a esta perspectiva Rodrigo, Rodríguez y Marrero (ob.cit), sostienen que:

...las teorías que constituye el hombre de la calle están implícitas, es decir, que no disponen de una formulación verbal sistemática y por tanto los argumentos que contiene son tácitos y sin especificar. Sin embargo, las teorías científicas, al basarse en las convenciones sociales de la conciencia, cuentan con una formulación verbal explícita y sus argumentos se estructuran lógicamente... (p.84)

El planteamiento anterior representa un ejemplo claro acerca de la construcción de teorías inconscientes por parte del hombre común en cualquier escenario. Un ejemplo lo constituye el ámbito educativo, donde los docentes construyen sus propias teorías implícitas, las cuales, a veces explicitan en sus accionar cotidiano.

Por ello, Benlloch (1997) plantea que los profesores sintetizan un conjunto de experiencias que configuran una estructura de conocimientos referidos a “...episodios o escenarios de intervención docente-discente que pasan a formar parte muchas veces del

Revista EDUCARE, Volumen 17, Número 3, Septiembre-Diciembre 2013. DEPOSITO LEGAL: ppi201002LA3674- ISSN: 2244-7296 (Revista Arbitrada e 
conjunto de creencias, conflictos, conocimientos personal, teorías personales o teorías implícitas..." (p.96). Esto es, el conocimiento que es "asumido o integrado como propio, auto atribuido...”; además, estos conocimientos inconscientes no sólo influyen en las preconcepciones que tienen los profesionales de la docencia sobre determinados fenómenos, sino que influyen también en las prácticas pedagógicas, acciones y decisiones de los docentes. Esto permite inferir la influencia directa que tienen estas concepciones implícitas en la manera como los educadores organizan las actividades de aula, la forma de seleccionar los métodos y técnicas de enseñanza, así como la introducción de los aspectos creativos como elementos integradores del currículo.

En este mismo orden de ideas, Rodrigo, Rodríguez y Marrero (ob.cit), afirman que el docente, como producto de su formación, ha construido significados de manera consciente. Pero, también otros que son el fruto “...de experiencias continuas y difusas sobre los más variados aspectos que podemos distinguir en un currículo: contenidos, destrezas, orientaciones metodologías, pautas de evaluación, etc.” (p.244), los cuales de forma inconsciente son almacenados en la memoria y difícilmente pueden ser expuestos de manera organizada y coherente. Asimismo, para los autores, las teorías implícitas en el campo educativo son “... teorías pedagógicas históricamente elaboradas y transmitidas a través de la formación y de la práctica pedagógica...” (p.244). Por tanto, es posible considerar estas teorías como un compendio de saberes culturales y de experiencias personales propias de cada docente, que guardan más relación con los complejos procesos de sus prácticas cotidianas, que con las teorías formales históricamente expuestas.

Como lo expresan Medina, Simancas y Garzón (1999) se plantea la necesidad imperiosa de examinar, minuciosamente, el pensamiento del profesional de la docencia, para comprender e interpretar los complejos procesos de enseñanza y aprendizaje que se desarrollan en los espacios escolares y a nivel de otros escenarios académicos.

Los planteamientos anteriores permiten introducir la tesis acerca de la posibilidad de explorar las teorías implícitas de determinados grupos sociales en cualquier dominio de conocimiento. Según Rodrigo, Rodríguez y Marrero (ob.cit) son muchos los términos, o temas de investigación, en los que se puede indagar acerca de las teorías implícitas. Para los efectos de la presente investigación, el dominio seleccionado fue el referido a la creatividad. 
Desde esta perspectiva, la investigación se centró en la exploración de las teorías implícitas sobre la creatividad de los docentes, en el área de didáctica, pertenecientes al Departamento de Formación Docente de la Universidad Pedagógica Experimental Libertador, Instituto Pedagógico de Barquisimeto (UPEL-IPB), por cuanto éstos son los encargados de introducir las innovaciones respecto al cómo enseñar, y de hacer operativo el currículo en todas sus dimensiones, inclusive la relacionada con la creatividad.

Ahora bien, se considera necesario describir e interpretar las teorías implícitas que poseen los docentes de didáctica acerca del dominio creatividad, por cuanto sobre ellos recae la responsabilidad de formar a los futuros docentes a la orden de los nuevos conocimientos científicos. Además, ello permitiría aportar información para realizar una práctica más consciente, en la medida en que puedan verbalizar y sistematizar sus conocimientos implícitos.

Del análisis realizado hasta aquí, surgen las siguientes inquietudes: ¿Qué teorías implícitas sobre la creatividad han construido los docentes de didáctica de la UPEL-IPB a lo largo de su devenir práctico?, ¿Cómo son las teorías implícitas de la creatividad construidas por los docentes de didáctica de la UPEL-IPB?, ¿Cuáles son los enunciados o frases típicas de cada teoría implícita de la creatividad construidas por los docentes de didáctica de la UPELIPB?, ¿Qué interpretaciones se pueden derivar de las teorías implícitas de la creatividad de los docentes de didáctica?

\section{Orientaciones del estudio.}

\section{Orientación General:}

Develar las teorías implícitas de la creatividad construidas por los docentes de didáctica de la UPEL-IPB a lo largo de su devenir práctico.

\section{Orientaciones Específicos}

Describir las teorías implícitas de la creatividad que tienen los docentes de didáctica de la UPEL-IPB.

Interpretar las teorías implícitas de la creatividad, de los docentes de didáctica, sobre la base de las teorías científicas históricamente expuestas.

Revista EDUCARE, Volumen 17, Número 3, Septiembre-Diciembre 2013. DEPOSITO LEGAL: ppi201002LA3674- ISSN: 2244-7296 (Revista Arbitrada e 


\section{ABORDAJE METODOLÓGICO.}

\section{Naturaleza del estudio.}

La presente investigación se inscribe en el episteme de la investigación social, específicamente en el paradigma socioconstruccionista, en virtud que se pretendió develar las teorías implícitas de los docentes de didáctica del Departamento de Formación Docente de la UPEL-IPB.

Según Sánchez de Varela y Márquez Pérez (2005), con el paradigma socioconstruccionista se busca “...revelar el significado de las formas particulares de vida social” (p.126). En este sentido, este estudio se orientó a revelar e interpretar las teorías implícitas de los docentes de didáctica adscritos al Departamento de Formación Docente de la UPEL-IPB, construidas durante interacciones sociales vividas en su formación académica y en el contexto en el que se desenvuelven.

Por esta razón, el proceso indagatorio es de naturaleza cualitativa, tal y como lo plantean Popper (citado en Martínez, 2004), por cuanto esta perspectiva “...trata de identificar, básicamente, la naturaleza profunda de las realidades, su estructura dinámica, como la posibilidad para dar razón plena del comportamiento y las manifestaciones de éstas” (p.44). Es así como el diseño de la investigación se orientó a identificar el comportamiento de los actores sociales en su cotidianidad, específicamente a la interpretación de las teorías implícitas de los docentes de didáctica del Departamento de Formación Docente de la UPELIPB.

Según, Denzin y Lincoln (citado en Boscán, 2003), el enfoque cualitativo permite estudiar la realidad en su contexto natural. Esto significó intentar dar sentido e interpretar los acontecimientos atendiendo a los propios significados que tuvieron para las personas implicadas y comprometidas con el procedimiento desarrollado. De ahí que, el análisis y la comprensión de las evidencias producidas durante el desarrollo de la investigación, se orientaron a develar el significado atribuido por los actores sociales, tanto individual como colectivamente.

Como se expresó previamente, la naturaleza es eminentemente cualitativa, por cuanto, según Martínez (ob.cit.), Taylor y Bogdan (1990), en su más amplio sentido, un proceso de indagación es cualitativo cuando se produce información descriptiva derivada de la expresión

Revista EDUCARE, Volumen 17, Número 3, Septiembre-Diciembre 2013. DEPOSITO LEGAL: ppi201002LA3674- ISSN: 2244-7296 (Revista Arbitrada e 
oral y escrita de los versionantes durante su participación en las interacciones intersubjetivas, así como el comportamiento observable durante situaciones de aula.

La experiencia vivida tuvo un carácter social, cognitivo y constructivista; social, por estar centradas en las personas, en las interacciones entre ellas y en el estudio del comportamiento humano (acciones). Cognitiva, puesto que partió de la concepción que se tiene del hombre sobre su capacidad de aprender, del procesamiento de la información y la construcción de teorías personales relacionadas con la creatividad. Y constructivista porque el estudio y análisis de las situaciones de aprendizaje se construyen en la realidad misma, con la interacción entre personas y grupos.

\section{El método.}

El método empleado fue el etnográfico, que según el planteamiento de Buendía, Colás y Hernández (1997) es la vía para conocer estudiar y describir determinados grupos sociales. Por tanto, las actividades intersubjetivas estuvieron centradas en el conocimiento de las teorías implícitas de actores sociales involucrados y comprometidos con la investigación. Además de ello, se usó como principal técnica de investigación la observación participante.

En este mismo orden de ideas, Martínez (ob.cit) plantea que la etnografía significa la “...descripción del estilo de vida de un grupo de personas habituadas a vivir juntas...” (p181.). Es así como, los docentes del Departamento de Formación Docente (UPEL-IPB) se constituyeron en los actores sociales que participaron en las actividades de producción de información cualitativa.

Asimismo, dadas las características de las personas participantes, según lo planteado por Buendía, Colás y Hernández (ob.cit), éste se ubica bajo la forma de microetnografía. Por cuanto estuvo centrado en observaciones e interpretaciones de los actores pertenecientes a una institución escolar, particularmente en la observación del comportamiento suscitado en un aula de clase. Aspecto que es acuñado por Martínez (ob.cit.), cuando establece que la unidad de análisis no sólo podría ser una nación, sino también un “...grupo humano que se constituya en una entidad cuyas relaciones estén reguladas por costumbres o por ciertos derechos y obligaciones” (p.182). De allí que el grupo de docentes seleccionados se configuró a partir de interrelaciones intersubjetivas, en la unidad de análisis del estudio en cuestión.

Finalmente, la adopción del método etnográfico requirió que el autor viviera largos períodos de tiempo junto a los docentes del Eje Didáctico para poder tener una imagen realista

Revista EDUCARE, Volumen 17, Número 3, Septiembre-Diciembre 2013. DEPOSITO LEGAL: ppi201002LA3674- ISSN: 2244-7296 (Revista Arbitrada e indizada) 
y fiel de las teorías implícitas que éstos manejan. De igual forma, ameritó largos períodos de toma de notas en los que se registró cada uno de los eventos, fenómenos, acciones, posturas, comentarios, entre otros, que fueron considerados relevantes para el proceso heurístico vivenciado.

\section{Abordaje práctico.}

El desarrollo de la investigación se llevó a cabo mediante la aplicación de un conjunto de técnicas de producción de información, entre las cuales se destacan; la observación participante, la entrevista en profundidad y los testimonios focalizados. Así como por el manejo y la utilización de algunos recursos (grabadoras, cuaderno de notas, entre otros) que permitieron producir evidencia cualitativa, de forma sistemática y directa, a partir de las palabras y el comportamiento de los versionantes comprometidos con los procesos enseñanza y aprendizaje.

Para el análisis de la información generada se recurrió al modelo GLATER, el cual permitió la sistematización e interpretación de la evidencia, modelo que según González de Flores y Hernández (2000) es:

...una herramienta útil para el procesamiento y organización de la evidencia cualitativa, el cual consiste en la organización de la información mediante tablas o matrices en función de los siguientes aspectos: a) Código o temática; b) Descripción; c) Categorías e; d) Interpretación... (p.23)

Una vez producida la información, se procedió a la elaboración de las tablas sugeridas por el modelo y a la interpretación de las categorías que emergieron en el análisis. Cabe destacar que el método GLATER fue complementado con los planteamientos esgrimidos por Rodrigo, Rodríguez y Marrero (1993), los cuales plantean en su metodología para la indagación de las teorías implícitas, un procedimiento que consiste básicamente en la selección de las frases típicas de cada teoría develada, que fueron producidas en las entrevistas y los otros medios de producción de información. Es decir se tomaron y se agruparon todas las frases afines a cada teoría y se organizaron mediante tablas, cada una de las cuales debía estar identificada con la teoría correspondiente.

Procedimiento que se realizó hasta agotar la evidencia producida. Esto ameritó varias revisiones de la evidencia y la clasificación o categorización de acuerdo con la teoría a la que correspondía cada frase. En consecuencia, el modelo GLATER permitió la sistematización e 
interpretación de la evidencia, mientras que la categorización se llevó a cabo según las recomendaciones de los teóricos que proponen la metodología para la exploración de las teorías implícitas, citados al inicio de este párrafo.

En cuanto al tema de la categorización, Martínez (ob.cit) apunta que este procedimiento de análisis no es más que “...clasificar la partes con relación a un todo” (p.266). Esto es, una vez producida la información necesaria para el desarrollo de la experiencia heurística se procedió a clasificar la evidencia de todas las fuentes de acuerdo con los aspectos comunes. Posteriormente, las evidencias agrupadas en grandes categorías fueron clasificadas en otras menos complejas denominadas subcategorías.

Seguidamente se realizó la triangulación de fuentes de generación de evidencias como: los registros de observación, de entrevistas y los testimonios focalizados, la cual permitió la contrastación de las frases típicas de cada teoría clasificada en el procedimiento anterior y la validación de las evidencias recogidas a través de las tres técnicas antes mencionadas.

Para Rodríguez, Gil y García (1999), la triangulación consiste en la integración y contrastación de la evidencia producida durante la investigación. A partir de este planteamiento se procedió a contrastar las frases típicas de cada teoría, seleccionadas de la información aportada por los docentes, informantes claves y la producida por el autor mediante la técnica de la observación participante.

La estrategia general de la investigación, y en especial el proceso de triangulación, hicieron posible precisar, describir e interpretar las teorías implícitas de la creatividad de los docentes de didáctica del Departamento de Formación Docente de la UPEL-IPB que de una u otra forma estuvieron relacionados con la investigación.

Para efectos de la investigación, y en función de los objetivos formulados, interesó observar el comportamiento de los docentes cuando orientaban su práctica de aula, y las expresiones orales, escritas y gestuales, entre otras.

\section{Técnicas e instrumentos.}

Las técnicas que se emplearon en esta investigación fueron: la observación participante, la entrevista en profundidad y los testimonios focalizados.

La observación participante consiste, según Rodríguez, Gil y García (1999), en “...la recogida de información que requiere una implicación del observador en acontecimientos o fenómenos que está observando” (p.165). Esta definición supuso que el investigador se

Revista EDUCARE, Volumen 17, Número 3, Septiembre-Diciembre 2013. DEPOSITO LEGAL: ppi201002LA3674- ISSN: 2244-7296 (Revista Arbitrada e indizada)

Página 14 
involucrara en cada uno de los acontecimientos vivenciados en las actividades de aula, conversaciones formales e informales que se desarrollaron a lo largo del estudio. Además de ello, implicó que el autor, a la vez que interactuaba con los actores sociales (que formaron parte de la investigación), mantuviera la atención en los diferentes aspectos que fueron reflejados en un papel de trabajo que sirvió de guía de observación. Donde el autor registró en su cuaderno de campo la información producida mediante la mencionada técnica.

En este mismo sentido, Buendía, Colás y Hernández (ob.cit) plantean que la técnica de la observación participante tiene como objetivo “...recoger datos de modo sistemático directamente de los contextos y situaciones específicas por lo que pasa el grupo” (p.269). Este planteamiento se llevó a cabo cuando el investigador se implicó en los acontecimientos vivenciados en las actividades de aula. Lo cual ameritó que el autor conviviera largos períodos de tiempo junto a los docentes, a la vez que se involucró en cada una de las experiencias realizadas.

Otra de las técnicas empleadas fue la entrevista en profundidad. Atendiendo al planteamiento de Martínez (ob.cit) se constituyó bajo la forma de un “...diálogo coloquial entre el investigador y los sujetos de la investigación” (p.87). Esta definición condujo a que el investigador estableciera una amplia conversación con los informantes claves. Aspecto que permitió a los actores sociales tener libertad parar expresar sus ideas y opiniones acerca de la creatividad.

Del mismo modo, este aspecto es acuñado por Rodríguez (ob.cit) cuando establece que el propósito de la entrevista en profundidad se orienta a indagar acerca de “...las ideas, creencias y supuestos mantenidos por otros” (p.169). Es así como la mencionada técnica llevó al investigador a indagar sobre las ideas y puntos de vista de los actores sociales comprometidos en el estudio.

También, en la adopción de la entrevista en profundidad, se dio importancia al planteamiento de Taylor y Bogdan (1987), quienes la definen como “...una conversación entre iguales” (p.101). En este caso, el investigador promovió un clima de confianza entre él y los informantes antes de las entrevistas, al punto de establecer el rapport necesario para iniciar la conversación.

Revista EDUCARE, Volumen 17, Número 3, Septiembre-Diciembre 2013. DEPOSITO LEGAL: ppi201002LA3674- ISSN: 2244-7296 (Revista Arbitrada e 
Para la realización de la entrevista en profundidad, se procedió a elaborar un papel de trabajo como guía de entrevista o guion temático. Luego se realizaron las entrevistas, después de la observación de las actividades de aula orientadas por los docentes de didáctica.

En cuanto a la aplicación de las entrevistas a profundidad, se puede destacar que éstas se hicieron de manera individual, luego de la observación y registro de las sesiones de aula en cada unidad de aprendizaje. Además, la mayoría de ellas fueron grabadas y luego transcritas en manuscritos. También, se cuidó la formulación de las preguntas y se evitó la rigidez en el orden de las mismas, a fin de poder identificar las teorías implícitas de la creatividad que manejan los actores sociales involucrados en la investigación.

En relación con los testimonios focalizados, éstos fueron concebidos, a partir del planteamiento de Briones (1998), como “...una técnica que consiste en solicitar a personas que han vivido determinadas experiencias las expresen mediante un testimonio escrito" (p.68). Eso es, los docentes de didáctica, a solicitud del investigador, procedieron a expresar, mediante una producción escrita, la experiencia vivida, en relación con la creatividad, durante procesos cotidianos y académicos. Una guía sirvió de orientación para la redacción del documento.

Por último, los instrumentos de investigación usados fueron: los registros en el diario de campo, el protocolo de conversación y las evidencias escritas como testimonios focalizados. Y entre los recursos, el cuaderno de campo y el grabador de audio.

\section{Actores sociales de la investigación.}

Los actores sociales del estudio fueron los docentes de didáctica adscritos al Departamento de Formación Docente de la UPEL-IPB. Estos docentes se desempeñan en el mencionado departamento administrando los cursos Estrategias para el Proceso EnseñanzaAprendizaje, Evaluación de los Aprendizajes y Planificación del Proceso EnseñanzaAprendizaje.

La selección de los participantes se sustentó en dos criterios fundamentales: el primero, que fuesen docentes del mencionado departamento, adscritos al Eje Didáctico, y el segundo, que manifestaran su voluntad de participar en la investigación. En otras palabras, la unidad de estudio estuvo conformada por los docentes del Eje Didáctico que de una u otra manera decidieron participar en el desarrollo de la investigación.

Revista EDUCARE, Volumen 17, Número 3, Septiembre-Diciembre 2013. DEPOSITO LEGAL: ppi201002LA3674- ISSN: 2244-7296 (Revista Arbitrada e 
El papel de los participantes en la investigación se centró en orientar las actividades de aula, normalmente con sus estudiantes, en función de los contenidos inherentes al curso que administraban durante el lapso académico. Mientras el investigador desarrollaba la observación participante y registraba la información producida, además de permitir la realización de las entrevistas y la producción de los testimonios focalizados.

\section{Escenario o espacio físico de la investigación.}

El espacio de estudio fue la Universidad Pedagógica Experimental Libertador, Instituto Pedagógico de Barquisimeto "Luís Beltrán Prieto Figueroa” ubicado en el Estado Lara, República Bolivariana de Venezuela. Este estuvo representado por un grupo de docentes del Departamento de Formación Docente, concretamente profesores del Eje Didáctico; de los cuales cinco (5) se desempeñaron como informantes clave.

Por otro lado, se contó con un espacio institucional integrado por otros actores: docentes y directivos que conocieron de la investigación. Estas personas, como el caso de la Jefa del Departamento de Formación Docente y otros colegas, contribuyeron con la facilitación de espacios físicos y la preparación de los materiales de estudio utilizados por los participantes de los cursos.

La selección de los docentes, que se desempeñaron como informantes claves, se sustentó en el criterio de que éstos interactuaban con el investigador desde hace varios años, y establecieron una relación personal y profesional positiva con él; a la vez que manifestaron su voluntad de suministrar la información necesaria. Aunque varios docentes presentaron tales características, para los efectos de esta investigación se procedió a seleccionar cinco (5) informantes claves.

Luego de realizar la observación participante de las actividades orientadas por los docentes en el aula de clases, los informantes suministraron información mediante sesiones de entrevistas en profundidad que se hicieron de modo individual. Esta actividad se desarrolló para cada unidad programática del curso.

Posteriormente, y una vez culminadas las sesiones de entrevistas, los informantes procedieron a elaborar un testimonio focalizado acerca de la experiencia vivida, personal y académica, relacionada con la creatividad. De manera que esta técnica permitió producir más información para complementar las expresadas de forma oral en las entrevistas.

Revista EDUCARE, Volumen 17, Número 3, Septiembre-Diciembre 2013. DEPOSITO LEGAL: ppi201002LA3674- ISSN: 2244-7296 (Revista Arbitrada e 


\section{LOS HALLAZGOS.}

El estudio de las teorías implícitas sobre la creatividad de los docentes de didáctica de la UPEL-IPB develó la presencia de cinco temáticas amplias, como son: Teoría de la creatividad como proceso (TCPRC), Teoría de la creatividad como ambiente (TCA), Teoría de la creatividad como producto (TCPRD), Teoría de la creatividad como persona (TCPER) y Teoría integral de la creatividad (TIC).

En relación con la Teoría de la creatividad como proceso (TCPR), se develaron cuatro (4) categorías que ilustran en su orientación hacia cuatro procesos distintos, que son: Teoría de la creatividad como proceso personal (TCPRCper.), Teoría de la creatividad como proceso didáctico (TCPRCdidác.), Teoría de la creatividad como proceso cognitivo (TCPRcog.) y Teoría de la creatividad como proceso paradójico (TCPRparad.).

En cuanto a la teoría de la creatividad como proceso personal (TCPRper.), se conoció que, muchas de las experiencias personales de los docentes de didáctica están relacionadas con la creatividad; o, en consecuencia, entienden que el hecho creativo está asociado a muchas de las experiencias vividas en los primeros años de vida, fundamentalmente en el contexto familiar. Esta teoría se develó cuando los actores sociales narraron situaciones de su niñez que relacionan con la creatividad. Por ejemplo, narraron experiencias asociadas al canto, el baile, la danza. Asimismo, expresaron experiencias vividas con docentes en su formación de pregrado que les permitió inspirar el camino hacia la práctica de la creatividad. Estas afirmaciones tienen asidero lógico en los siguientes actos de habla enunciados por los versionantes:

“...David Vivas es para mi una persona inspiradora para desarrollar este proceso de creatividad..."

"Las experiencias sociales, personales, la Profesora Miriam Márquez...es una persona que para mi inspiró ese camino...”

“...de mi niñez recuerdo que disfrutaba muchísimo lo que hacia, me encantaba estar en actividades relacionadas con la creatividad, por ejemplo el valet, el baile, la danza..."

También, para el caso de la creatividad como proceso didáctico (TCPRdidác.), se develó que el hecho creativo está asociado a las actividades relacionadas con la enseñanza y el aprendizaje. En el que los métodos y técnicas creativas tienen una posición preponderante para los docentes comprometidos con el estudio. Esto puede ilustrase cuando los docentes refirieron el uso de técnicas como: mapas mentales, mapas conceptuales, ideograma, juego,

Revista EDUCARE, Volumen 17, Número 3, Septiembre-Diciembre 2013. DEPOSITO LEGAL: ppi201002LA3674- ISSN: 2244-7296 (Revista Arbitrada e indizada) 
mímica, dramatización, teatro, entre otros. Estos planteamientos tienen apoyo empírico en los siguientes enunciados generados por los actores sociales mediante las técnicas de investigación cualitativa:

"Para la exposición deben usar procesadores como mapas mentales, caricaturas, mapa conceptuales..."

"No olviden que la semana que viene vamos a trabajar con mapas conceptuales e ideogramas...”.

“...esa experiencia con el trabajo de recreación donde uno tiene que trabajar la mímica, la música...”

Por su parte, la teoría de la creatividad como proceso cognitivo (TCPRcog.) se manifestó como hecho ligado a la cognición de la persona, representado mediante la actividad de pensamiento que el sujeto tendrá que poner en práctica para la producción de respuestas originales y novedosas. No obstante, se develó igualmente la influencia que puede tener el docente en la activación o desarrollo de estos procesos, hasta el punto de poder generar barreras que los coarten. Estas posturas encuentran sentido en algunas frases emitidas por los profesores de didáctica, en las cuales explicitan que del docente depende, mediante la práctica de la creatividad, la activación del pensamiento para la producción de nuevas ideas, o, de lo contrario, el bloqueo de las mismas. Estas ideas pueden apreciarse en las siguientes frases típicas expresadas por los docentes durante el proceso investigativo:

“...porque de repente yo concibo que pudieran emerger procesos creativos...”

“...la gente cree que los procesos creativos se llevan a cabo de manera desordenada y no es así...”

“...eso me llevo a autoevaluarme...en aras de no frenar el proceso creativo en los alumnos...”

De igual modo que las categorías anteriores, la teoría de la creatividad como proceso paradójico (TCPRparad.) se conoció en el análisis presentado por la generación de respuestas originales e ingeniosas frente a situaciones difíciles o de conflicto, en el plano personal, pero también en el plano laboral. Como se puede apreciar, esta categoría constituye una situación bastante interesante, porque muchos se han preguntado ¿cómo es que se puede ser creativo en momentos difíciles? Pero, los docentes de didáctica consideran que sí se puede ser creativo en situaciones adversas; o, lo que puede ser lo mismo, las situaciones de conflicto llevan a generar nuevas respuestas, originales e ingeniosas. Esta afirmación se develó en el momento en que los docentes expresaron algunas proposiciones que así lo corroboran. Por ejemplo, en

Revista EDUCARE, Volumen 17, Número 3, Septiembre-Diciembre 2013. DEPOSITO LEGAL: ppi201002LA3674- ISSN: 2244-7296 (Revista Arbitrada e 
algunos fragmentos de entrevista, los docentes narraron situaciones que requirieron ser creativos para poder enfrentarlas, como: la muerte de un familiar, el quedar solas criando a sus hijos (en el caso de las damas). Lo anterior tiene sustento práctico en las siguientes expresiones de los actores sociales comprometidos con la investigación:

“...tu puedes ser creativo hasta en los momentos más difíciles, que no tienes todos los ingredientes para hacer un plato particular, pues, tienes que ingeniártelas y sale algo, y sale muy bien..."

“...buscar la armonía en situaciones que se presentan como situaciones de conflicto...”

“...el haber criado sola a mis hijos a temprana edad, yo creo que eso fue un malabarismo, es otro episodio bien impactante que me marcó...”

Por otro lado, la Teoría de la creatividad como ambiente (TCA), se develó en dos (2) categorías con dos sentidos distintos. Ellas son: la teoría de la creatividad como ambiente físico (TCAfísico), y la teoría de la creatividad como ambiente psicológico (TCApsicol.).

Para el caso de la TCAfisico, se precisó se caracteriza por la interacción del sujeto con elementos materiales del entorno que le permiten generar ideas o respuestas igualmente novedosas e ingeniosas, pero cuyos elementos materiales se encuentran más asociados a los recursos o herramientas que utilizan durante el proceso didáctico. Esto lleva a pensar que la disposición de estos recursos materiales configura un escenario propicio para el desarrollo de la creatividad. Esto lo ilustran las expresiones verbales de los docentes tomadas de las la evidencia en las que se destacan recursos como: juegos, libros, salas de video, entre otros; que según los versionantes demandaron la activación de su creatividad al momento de la interacción con éstos. Ello se ve reflejado en los actos comunicativos referidos por los informantes clave durante los procesos de interacción intersubjetivas vivenciados en la investigación que se indican a continuación:

“...los medios didácticos que se utiliza, esos también intervienen dentro del proceso...en la medida de que el profesor le da dinamismo al proceso, presente nuevas herramientas...”

“...cuál es el contexto en el que te vas a desempeñar, con qué cuentas, qué herramientas tienes a tu disposición...”

“...el ambiente, cómo organizarlo. Siempre fue distinto al resto de los profesores...”

Seguidamente, para el caso de la TCApsicol., se interpretó que la creatividad es la consecuencia de generar un clima psicológico de libertad y tranquilidad, en el que el sujeto

Revista EDUCARE, Volumen 17, Número 3, Septiembre-Diciembre 2013. DEPOSITO LEGAL: ppi201002LA3674- ISSN: 2244-7296 (Revista Arbitrada e 
puede tener la oportunidad de opinar, producir y generar ideas nuevas e ingeniosas. No obstante, este clima bien pudiera generar barreras que coarten o inhiban los procesos creativos. De allí la importancia de que el docente mediante su práctica induce la constitución de un ambiente propicio para la generación de ideas o productos creativos dentro del aula de clases. Estas premisas tienen consonancia con expresiones producidas durante la investigación, en las que los docentes manifestaron que debe existir libertad para emitir opiniones. Como también se debe generar un clima de confianza y un clima flexible para que el estudiante aprenda. Aspectos que se ven ilustrados en las siguientes frases características de esta teoría:

“...un estudiante que está amenazado, pues, cerebralmente su reptil o su subsistema cerebral reptil lo va a poner a la defensiva...”

“...ese ambiente de aprendizaje...debe ser un ambiente de aprendizaje con flexibilidad en el cual exista libertad para expresar opiniones...”

“...debe haber un ambiente de libertad más no de libertinaje, pero si libertad...”

En la misma dirección que la teoría anterior, en la teoría de la creatividad como producto (TCPRD) se evidenciaron dos (2) categorías representativas que son: Teoría de la creatividad como producto tangible (TCPRDtang.), la Teoría de la creatividad como producto abstracto (TCPRDasbt.).

La primera subcategoría, TCPRDtang., refiere que la creatividad es la capacidad de la persona para generar un producto u objeto tangible, el cual debe generar un impacto en el contexto donde se produce. Además, debe exhibir ciertas características que lo acreditan como un elemento ingenioso y novedoso. Para el caso de los docentes de didáctica, refirieron productos que pueden servir como recursos empleados en los procesos enseñanza y aprendizaje; entre los cuales se destacaron: libros, poemarios, juegos y periódicos. Estas aseveraciones encuentran sustento en estos enunciados:

“...el alumno se ha planteado como trabajo fundamental que va a hacer un poemario...”

“...a través de los productos que el alumno decida elaborar..."

"...hace rato te mostré el libro que me publicaron que gira en torno a la creatividad...”

En la segunda categoría, denominada TCPRDabst., se concibe la creatividad como la capacidad de la persona para generar ideas o respuestas ingeniosas y originales frente a diversas situaciones. Esta afirmación tiene correlación con algunos actos de habla extraídos de

Revista EDUCARE, Volumen 17, Número 3, Septiembre-Diciembre 2013. DEPOSITO LEGAL: ppi201002LA3674- ISSN: 2244-7296 (Revista Arbitrada e 
la información producida durante el estudio, en los cuales los docentes expresaron la importancia de resolver problemas de la vida cotidiana y escolar sin apelar a un objeto tangible, sino mediantes respuestas o acciones ingeniosas a situaciones de conflicto. Por ejemplo, los versionantes narraron episodios de la vida diaria en la que fue necesario tomar decisiones rápidas para dar respuesta a dificultades de forma oportuna e ingeniosa, sin tener que elaborar o manipular un instrumento material. En este caso el producto generado se tradujo en una decisión, acción, respuesta, entre otras, que permitió salir adelante al inconveniente. Esto lo develan las siguientes frases tomadas de la evidencia:

“...producir y comunicar nuevas ideas. Como docente permanentemente tienes que estártelas ingeniándotelas para saber cómo enseñar y para saber cómo los estudiantes aprenden...”

“...tienes que estar produciendo ideas, pensando...generar nuevas ideas sobre cómo se mejora la acción pedagógica en el aula...”

“...el generar una ruta de cómo yo asumo mi formación, es un acto creativo...”

Para el caso referido a la Teoría de la creatividad ligada a la persona (TCPER), se develó la presencia de dos (2) categorías representativas de la misma. Ellas son: Teoría de la creatividad como potencialidad o capacidad de la persona (TCPERpotenc.), y Teoría de la creatividad como cualidad humana (TCPERcualid.).

En el primer caso, TCPERpotenc., la creatividad se vislumbra como la capacidad innata de las persona de realizar actos creativos. En este sentido, dada las experiencias a las que pueden ser sometidos los individuos, ésta puede configurarse en una habilidad. Además de ello, esta característica humana de crear puede aflorar en muchas en las diferentes actividades que realiza la persona. Por ejemplo, se puede manifestar creatividad al escribir, dibujar, hacia la música, entre otros. Asimismo, se conoció que los docentes de didáctica entienden que la realización de actos creativos innatos se desarrolla a partir de las experiencias que propicia el docente en los ambientes de aprendizaje. Ello puede apreciarse en las frases que se indican a continuación que así lo ilustran:

“...porque lo develó el diagnóstico, que tiene potencialidades al escribir...”

“...desde el punto de vista de las potencialidades creativas del ser humano...”

“...de repente para el otro es un atropello a las potencialidades creativas..."

La segunda categoría de la teoría TCPER, TCPERcualid., refiere que la creatividad es una cualidad inherente al ser humano como tal. Esto es, la creatividad está asociada a una serie de cualidades propias del ser humano, entre las que se destacan: capacidad de establecer 
relaciones interpersonales; de manifestar ternura, inconformidad permanente, sentimientos, posicionamiento ante la vida, curiosidad, apertura al cambio, entre otras. Es así como lo interpretado tiene consonancia con lo expresado por los docentes de didáctica durante procesos de interacción comunicativa:

“...la ternura, la ternura implica un acto creativo, pero entender que tú eres un ser humano, y eso forma parte de ti..."

“...eso también me ha permitido ver las cosas de manera distinta y asumir la creatividad desde el plano personal...”

"La creatividad...tiene que ver con elemento del sentir, es muy humano. Yo creo que tiene características de sublime...”

Finalmente, se develó la Teoría integral de la creatividad, la cual integra elementos de las teorías discutidas anteriormente. En ésta la creatividad es concebida como la combinación de elementos o factores que tienen que ver con las potencialidades y cualidades de la persona, con el aprovechamiento de las herramientas que ofrecen los ambientes o el contexto donde se produce el acto creativo y la activación de un sinnúmero de procesos que desencadenan en la generación de productos inéditos y novedosos. Ello tiene sustento empírico en la siguiente expresión formulada por los versionantes:

“...la creatividad es...el aprovechamiento total de todas las herramientas que generen en procesos innovadores en aras de la formación integral del ser y el logro pleno del hombre...”.

\section{REFLEXIONES FINALES.}

Toda persona puede construir sus propias teorías a partir de sus interacciones sociales y con el medio en general que le rodea, por cuanto cognitivamente está provista de ciertas capacidades que así lo permiten. Estas teorías implícitas se guardan en la mente de la persona de forma inconsciente, aspecto que dificulta que puedan ser verbalizadas coherentemente por el propio sujeto.

La mayoría de las teorías implícitas son representaciones mentales de naturaleza icónica, episódica y conjetural; muy pocas veces se alojan en la mente del sujeto como contenido semántico. Además de ello, son de carácter individual y se originan a partir de la asociación de experiencias o episodios vivenciados por las personas con los esquemas existentes de forma inconsciente, con la intención de dar explicaciones a ciertos fenómenos del medio que lo rodea. Asimismo, difícilmente el sujeto puede hacer consciencia de sus

Revista EDUCARE, Volumen 17, Número 3, Septiembre-Diciembre 2013. DEPOSITO LEGAL: ppi201002LA3674- ISSN: 2244-7296 (Revista Arbitrada e 
teorías implícitas. Esto puede ser posible sólo con la ayuda de otras personas. Por tanto, es de suma importancia generar espacios de interacción intersubjetivas donde el educador tenga la oportunidad de echar una mirada hacia sus significados ocultos y poder avizorar el carácter erróneo de estas teorías. También, es preciso resaltar para emprender cambios o transformaciones educativas que deben pasar por la revisar el pensamiento del docentes, es necesario partir del conocimiento que de forma inconsciente éste ha construido en el devenir de su experiencia profesional.

El ser humano, entendido como la persona común u hombre de la calle que lo diferencia del científico, puede construir teorías implícitas en diferentes dominios de conocimiento. Esto es, la persona, de acuerdo con el escenario o realidad donde se desenvuelve, es capaz de construir teorías en diferentes ámbitos de la vida; y en diferentes dominios de conocimiento.

En cuanto al docente, se tiene que construye teorías sobre aspectos de la educación (como las concepciones de la enseñanza, la evaluación, estrategias, los contenidos en diversas áreas del conocimiento), que no les resultan fáciles de describir o explicitar de forma coherente y sistematizada.

Estos conocimientos, construidos por los docentes, redundan en la forma como programan y orientan la enseñanza a nivel de los espacios de aprendizaje. Es decir, la está condicionada por sus teorías implícitas. En consecuencia, la exploración de estas teorías se torna un tema bastante interesante, por cuanto ello debe desembocar en la generación de cambios en los programas de formación del profesorado y en el mejoramiento de la práctica de la enseñanza. En este sentido, los esfuerzos que se realicen en investigación deben orientarse a la exploración de los significados ocultos y el grado de congruencia de éstos con las teorías formales que actualmente se tienen sobre los diversos dominios de conocimiento relativos a la práctica docente.

Los docentes de didáctica de la UPEL-IPB han construido inconscientemente sus propias teorías sobre la creatividad, con las cuales interpretan y dan sentido a su práctica pedagógica. Estas teorías netamente son episódicas, por cuanto fueron develadas durante la narración de experiencias vividas en su accionar en diferentes ámbitos de la vida cotidiana. Es por ello que, esta experiencia resulta una alternativa valiosa en tanto que permite introducir la posibilidad que tienen todos los docentes de este departamento y el resto de la UPEL de crear

Revista EDUCARE, Volumen 17, Número 3, Septiembre-Diciembre 2013. DEPOSITO LEGAL: ppi201002LA3674- ISSN: 2244-7296 (Revista Arbitrada e 
espacios de interacción que permitan la sistematización de las teorías implícitas. No solo por el carácter erróneo que éstas tengan sino por lo significativo y pertinentes que éstas puedan ser como conocimiento alternativo.

Las teorías implícitas develadas representan un conjunto de representaciones mentales bien significativas ya que se configuran en un bagaje de conocimientos valiosos para la comprensión de los procesos relacionados con la creatividad, la enseñanza y el aprendizaje; además por la significación que tienen como conocimiento relevante y alternativo construidos en escenarios educativos reales mediante la experiencia intersubjetivas entre pares.

En consecuencia, este nutrido repertorio de conocimientos, representativos de las teorías implícitas, sobre la creatividad puede ser fuente de inspiración en la generación de cambios en los programas de formación del profesorado, y para el mejoramiento de la práctica de la enseñanza y el aprendizaje. Más aún, cuando en estos tiempos se habla de cambios curriculares en el sistema educativo venezolano.

\section{REFERENCIAS.}

Argyris, C. y Schön, D. (1978). Organizational Learning: A Theory of Action Perpective C.A. Chicago: The Dryden Press.

Benlloch, M. (1997). Desarrollo cognitivo y teorías implícitas en el aprendizaje de las ciencias. España: Visor.

Bolívar, A. (2000). Los centros educativos como organizaciones que aprenden. Madrid: La muralla.

Boscán, A. (2003). El saber pedagógico del docente. "Una reconstrucción biográfica”. Tesis de doctorado no publicada, Universidad Nacional Experimental Simón Rodríguez, Barquisimeto.

Buendía, L., Colás, M. y Hernández, F. (1997). Métodos de investigación en psicopedagogía. España: McGraw-Hill.

Briones, G. (1998). Módulo curso de educación a distancia. Caracas: IPFESS-PILE.

Gonzalez de Flores, G. y Hernández, T. (2000). Análisis e interpretación de la información en la investigación cualitativa. Barquisimeto: Horizonte.

Medina, A., Simancas, K. y Garzón, C. (1999). El pensamiento de los profesores universitarios en torno a la enseñanza y demás procesos implícitos. Revista Electrónica Interamericana de Formación del Profesorado. (p. 513-569).

Martínez, M. (2004). Ciencia y arte en la metodología cualitativa. México: Trillas.

Porlán, R. y Rivero, A. (1998). El conocimiento de los profesores. España: Diada.

Revista EDUCARE, Volumen 17, Número 3, Septiembre-Diciembre 2013. DEPOSITO LEGAL: ppi201002LA3674- ISSN: 2244-7296 (Revista Arbitrada e 
Porlán, R. (1996). Cambiar la escuela. Argentina: Ministerio del Río de la Plata.

Reyes, L., Salcedo, E. y Perafán, G. (1999). Acciones y creencias. Bogotá: Universidad Pedagógica Nacional.

Rodríguez, G., Gil, J. y García, E. (1999). Metodología de la investigación cualitativa. España: Aljibe.

Rodrigo, M., Rodríguez, A. y Marrero, J. (1993). Las teorías implícitas: una aproximación al conocimiento cotidiano. España: Visor.

Sánchez de Varela, F. y Márquez Pérez, E. (2005). Criterios para la orientación de la evaluación de proyectos de investigación, trabajos de grado de especialización, trabajos de grado de maestría y tesis doctorales de naturaleza cualitativa. Revista Educare, Volumen 9(3), p. 123-136, Octubre 2005, Barquisimeto.

Taylor, S. y Bogdan, R. (1990). Introducción a los métodos cualitativos de investigación. La búsqueda de significados. Buenos Aires: Paidos. 